Signal \& Image Processing : An International Journal (SIPIJ) Vol.8, No.4, August 2017

\title{
COMPRESSION BASED FACE RECOGNITION USING TRANSFORM DOMAIN FEATURES FUSED AT MATCHING LEVEL
}

\author{
Srinivas Halvi ${ }^{\mathrm{a}}$, Nayina Ramapur ${ }^{\mathrm{b}}, \mathrm{K}^{\mathrm{B}} \mathrm{Raja}^{\mathrm{c}}$ and Shanti Prasad ${ }^{\mathrm{d}}$ \\ ${ }^{a}$ Dayananda Sagar College of Engineering, Bangalore, India. \\ ${ }^{b}$ Sai-Tektronix Pvt. Ltd., Bangalore, India. \\ ${ }^{c}$ University Visvesvaraya College of Engineering, Bangalore, India. \\ ${ }^{\mathrm{d}}$ K.S. Institute of Technology, Bangalore, India.
}

\begin{abstract}
The physiological biometric trait face images are used to identify a person effectively. In this paper, we propose compression based face recognition using transform domain features fused at matching level. The $2 D$ images are converted into 1-D vectors using mean to compress number of pixels. The Fast Fourier Transform (FFT) and Discrete Wavelet Transform (DWT) are used to extract features. The low and high frequency coefficients of DWT are concatenated to obtained final DWT features. The performance parameters are computed by comparing database and test image features of FFT and DWT using Euclidian Distance (ED). The performance parameters of FFT and DWT are fused at matching level to obtain better results. It is observed that the performance of proposed method is better than the existing methods.
\end{abstract}

\section{KEYWORDS}

Biometrics, Face Recognition, FFT, DWT, fusion Technique

\section{INTRODUCTION}

The biometrics is unique way of identifying humans based on physical and behavioral characteristics. The biometric is derived from Greek word bio i.e., life and metric i.e., measure, which is used to measure the human characteristics to authenticate a person. The biometrics is classified into two group's viz., physiological and behavioral biometrics. The physiological biometrics are related to the parts of human body and normally do not vary in the life time. The example of physiological biometrics are face, fingerprint, DNA, iris, Hand geometry etc. The behavioral biometrics are related to the behavior of a person and the characteristics are varied based on mood of a person and circumstances. The examples are signature, gait, key stroke, voice etc. The biometric system works in two modes viz., (i) Verification mode in which one to one comparison of biometric features. The captured biometric trait is compared with a specific biometric trait stored in a biometric database in order to validate a person to be claimed. (ii) Identification mode in which one to many comparisons of biometrics. The captured biometric trait is compared with many biometric samples stored in the biometric database in order to establish the identity of an unknown person. The face is a powerful biometric trait to recognize a person, as the face image can be captured without any cooperation of humans. The challenges in the face 
Signal \& Image Processing : An International Journal (SIPIJ) Vol.8, No.4, August 2017

recognition are pose verifications, illumination variations, expressions, occlusions, age factors, gender factors etc. The biometrics are used in banks, logical access control, health care, cloud computing, consumer applications, Border control, Airports, Biometric locks, automobiles, justice and low enforcement etc.

Contribution: In this paper, Compression based Face Recognition using Transform Domain Features fused at matching level is proposed. The face images are converted into 1D transform domains using FFT and DWTs. The performance parameters are computed by comparing features of database and test face images. The performance parameters are fused at matching level to obtain better results.

Organization: The remaining section cover literature survey of recent research papers. Section 3 presents proposed method and algorithm is given in Section 4. Section 5 explains performance analysis of proposed method. The conclusion is given in section 6

\section{LITERATURE SURVEY}

In this section, the existing techniques of feature extraction based on spatial and transformed domain methods are explained. The preprocessing techniques to modify original face images and classifiers to identify face images are also described. Ramy C.G.Chehata, et al.,[1] proposed face recognition method using transform domain techniques on the MPCA. The techniques are two dimensional and diagonal modular PCA. The diagonal modular PCA provides more background information of the face image and it is applied on both overlapping and non-overlapping blocks. Md. IqbalQuraishi, et al., [2] presented a face recognition method using Artificial Neural Network. In this method the ripplet transform is used for feature extraction, after preprocess and image enhancement of the face image.

Jing Wang, et al., [3] have proposed method that uses both the sparsity and correlation. Previously many papers suggested that the sparsity representation based classification (SRC) gives the improved performance. The sparsity is useful for selecting the sample and the correlation is better for comparing the query image and the training samples. Zhao-Rong Lai, et al., [4] developed a robust method for face recognition using discriminative and compact coding. In this method two sets of coding models are used, viz., Discriminative Coding (DC) and Compact Coding (CC). The DC gives the distinguishing decisions and gives the multi-scale error measurements where as the $\mathrm{CC}$ is useful for the face images with different illuminations. The proposed method combines both DC and CC to perform classification.

Dmitry Gorodnichy and Eric Granger [5] introduced the target based evaluation of face recognition technology for video surveillance applications. In this two methods are mentioned Cohert based and Target based methodologies for Watch List Screening (WLS). The proposed method is evaluated for the choke post public dataset. If the person in the video surveillance matches with the criminal dataset then the system makes an alarm. Compared to the both above mentioned techniques the target based method is suitable for the WLS. Yi Jin, et al., [6] proposed a method for identifying heterogeneous face recognition which contains different types of face dataset. Coupled Discriminative Feature Learning (CDFL) method is used to recognize the face for heterogeneous database. In the training session the CDFL seeks to provide most favorable filters to discriminative face recognition. The subjects with same shape indicates same person. 
Signal \& Image Processing : An International Journal (SIPIJ) Vol.8, No.4, August 2017

Asem M. Ali, [7] proposed a method of 3D-Based Pose Invariant Face Recognition at a Distance Framework for 3D faces recognition. In this method the face image acquisition camera is used. The captured face image features are extracted and given as input to the second camera, which will capture the faces of the subject in different orientation. Each face images features are extracted then $3 \mathrm{D}$ reconstruction performed after cropping. The test image is also followed the same procedure and the bio-signature of test image is obtained. PriyankaDharani and A S Vibhute [8] proposed a method face recognition technique using wavelet and Neural Network. The DWT is used to extract features. The Principal Component Analysis is also used along with Fisher Linear Discriminant to obtain final features. The feed forward neural network is used as classifier to identify face images. Lihong Wan, et al., [9] proposed face recognition method using convolutional neural network and subspace learning. The proposed method employs the deep learning of convolutional neural network. For the dimensionality reduction, two methods are used namely linear discriminate analysis (LDA) and whitening principal component analysis (WPCA). The method is tested using two face databases CMU PIE and FERET.

PawanpreetKaur and Deepak Aggarwal [10] proposed a face recognition method using DWT, LBP and PCA. For the first level feature extraction, DWT and LBP are used separately then PCA is applied on the fusion of LBP and DWT. For the comparison K-nearest neighbor (KNN) classifier is used. Kumuda and Basavraj[11] proposed an algorithm to extracting the better features of text images from complex scene images using Median filtering, Connected Components, DWT and Morphological operations. Hameed R. Farhan et al, [12] proposed a face recognition based on discrete HMM which is used to increases the recognition rate in order to make strong. The concept of novel start with preprocessing to filtering the image with median filter and DWT. The DWT used to compress the size of input image prepared to generate a sequence of overlapping blocks. The quantization method is converting each blocks to an integer value. The $100 \%$ recognition rate is achieved with reducing number of stats of HMM.

\section{PROPOSED MODEL}

In this section compression version face recognition based on transform domain features fused at matching level is proposed. The proposed face recognition model is shown in the figure 1 . The mean is applied on the face images to convert 2- Dimension (2D) into 1-Dimension (1D) to get the compressed feature which will provide appropriate features.

The Fast Fourier Transform (FFT) and Discrete Wavelet Transforms (DWT) are applied to obtain transform domain features. The low frequency coefficients obtained using DWT implies significant information and the corresponding values are high. The high frequency coefficients of DWT gives detailed information of the signals and the corresponding values are low ie., may be zeroes. The log is applied on high frequency coefficients to convert very low values to moderately high values. The low and converted high frequency values are concatenated to obtained final DWT features. The features of FFT and DWT are compared separately using ED to compute Performance Parameters. The fusion formula is used to combine results of FFT and DWT to obtain better results. 
Signal \& Image Processing : An International Journal (SIPIJ) Vol.8, No.4, August 2017

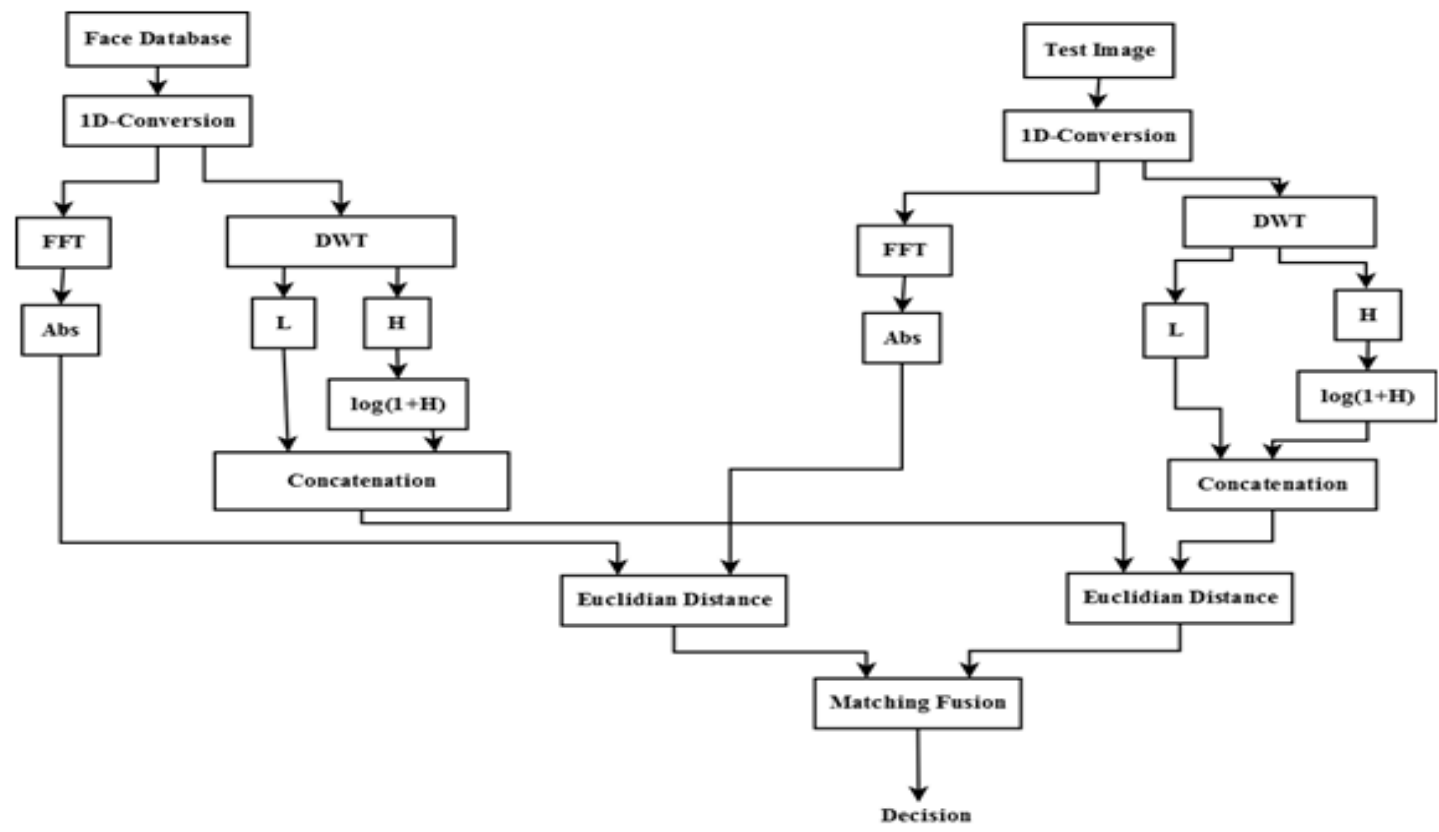

Fig. 1: Block Diagram of Proposed Model

3.1 Face Databases: The face recognition model is tested for various performance parameters using available face databases such as Olivetti Research Laboratory (ORL), Near Infra Red (NIR), Yale, Indian Male and Indian Female.

3.1.1) ORL Face database [13]: The Database contains totally 40 subjects. For each subject ten different orientated face images are captured. The resolution of an image is $112 * 92$. The samples of one person are shown in figure 2 .
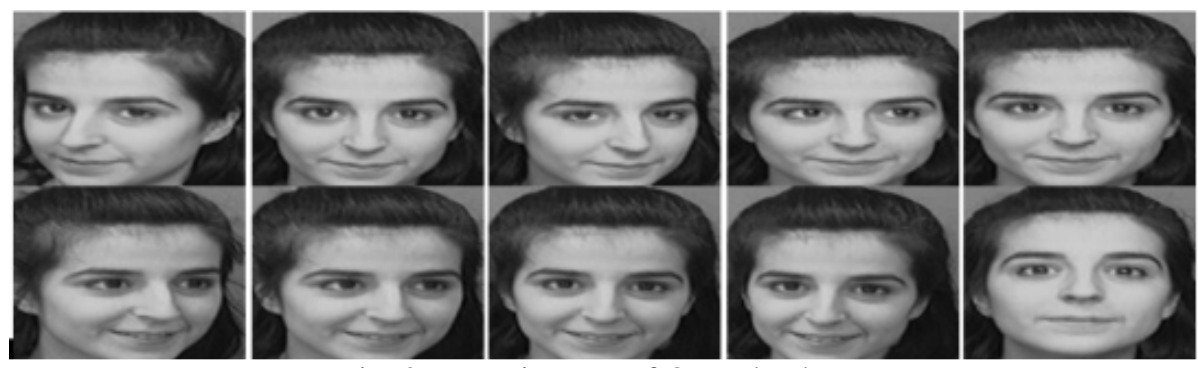

Fig. 2. Face images of ORL database

3.1.2) NIR Database [14]: This database created by considering less luminance intensity. The resolution of image is $576^{*} 768$. The database consists of 120 persons. Each person has fifteen face images with different orientations, with and without glasses. The samples of one person is shown in figure 3. 
Signal \& Image Processing : An International Journal (SIPIJ) Vol.8, No.4, August 2017

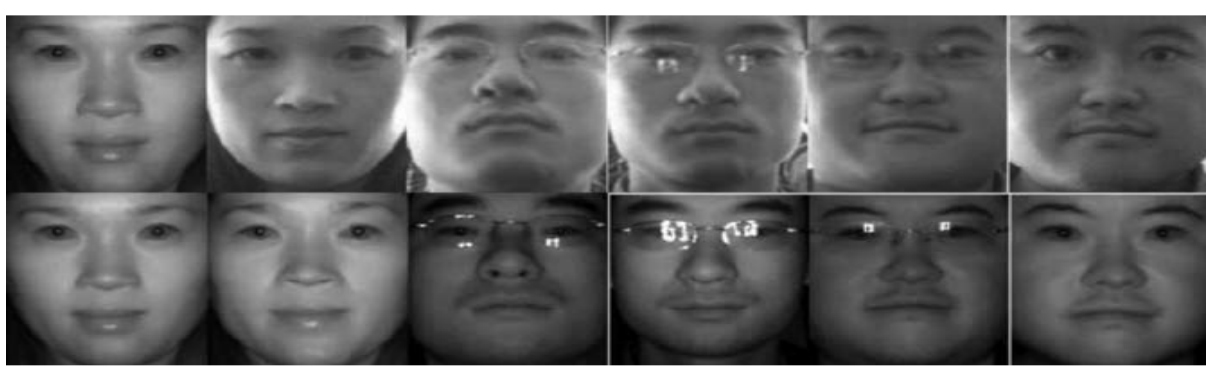

Fig. 3. Face images of NIR Database

3.1.3) YALE Database [15]: The database consists of 15 persons. Each person has ten different oriented images with resolution of 240x320. The sample of database image is shown in figure 4 .

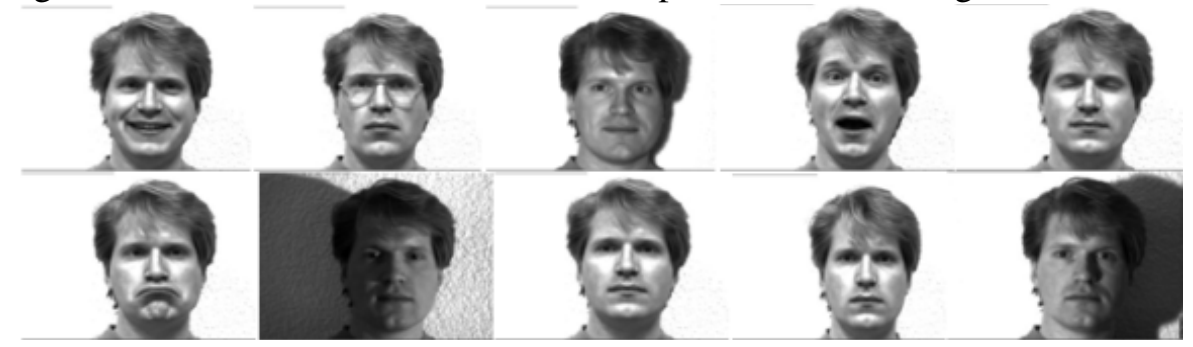

Fig. 4. Yale Database samples

3.1.4) Indian Male Database [16]: The database consists of twenty persons. Each person has eleven images with pose and expression variations having a resolution of 640x480. The samples of database face image are shown in figure 5.

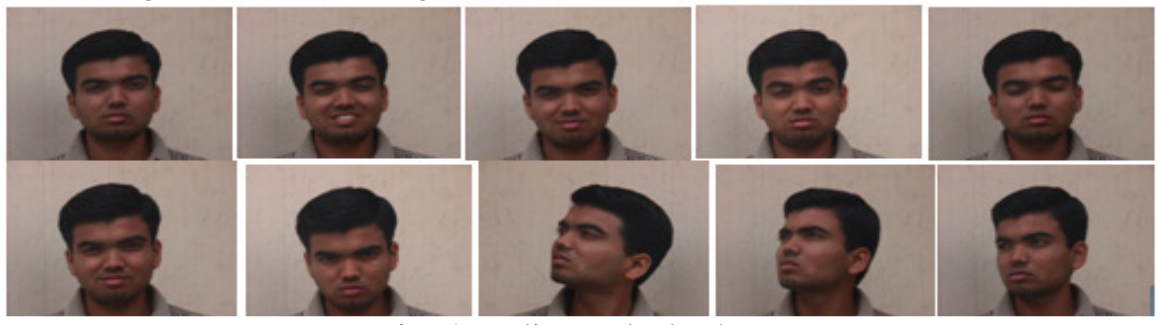

Fig. 5. Indian male database

3.1.5) Indian Female Database [17]: The database consists of twenty two persons with eleven images per person. The images are captured using a camera with a resolution 640X480. The face images are captured with different facial expressions and poses. The samples of database are shown in figure 6.

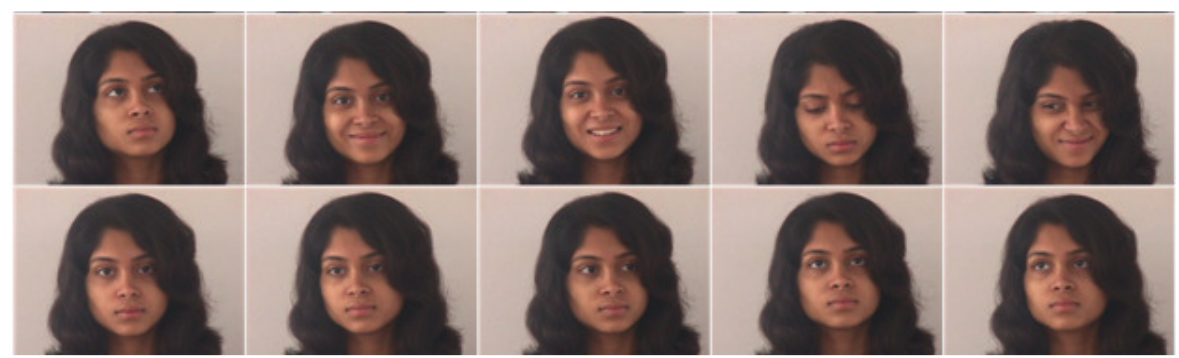

Fig. 6. Indian female sample images 
Signal \& Image Processing : An International Journal (SIPIJ) Vol.8, No.4, August 2017

3.2 1D-Conversion: The 2D image is converted to $1 \mathrm{D}$ column vector using mean computation on rows of image matrix. The advantage of converting $2 \mathrm{D}$ to $1 \mathrm{D}$ is that the number of pixels in an image decreases, which results in less computation time and memory requirement. The illustration of converting 2D matrix into 1D column vector is as shown in figure 7.

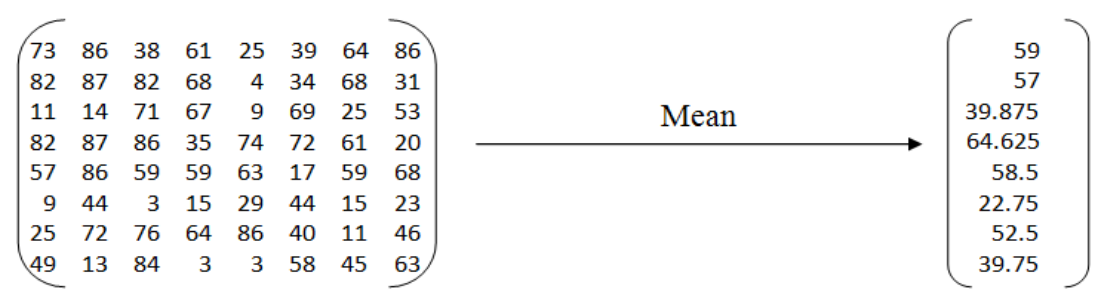

Fig. 7 Conversion of 2D matrix to 1D Column Vector

The $8 \times 8$ matrix is considered and mean is used on each row to convert row vector into scalar value. The column vector has every information of $2 \mathrm{D}$ matrix in a compressed manner. The computation time to convert 1D column vector into FFT and DWT domains are fast compared to 2D-FFT and 2D-DWT conversion.

3.3 Fast Fourier Transform Features [18]: The transformation is applied on 1D column vector to convert time domain into frequency domain. The transform decomposes vector into its real and imaginary components which is a representation of the vector in the frequency domain. The FFT of 1D signal given by the equations 1 .

$X(k)=\sum_{n=0}^{N-1} x(n) e^{\frac{-j 2 \pi k n}{N}}$

Where $\mathrm{N}$ is the number of samples.

The computed features are complex variables and the final features considered as absolute values.

3.4 Discrete Wavelet Transform features [19]: This gives information of the frequency variation along with the localization i.e., the time at which the frequency is varied. The DWT applied on Signal or 1D array gives two bands L and $\mathrm{H}$ i.e., approximate and detail coefficients respectively. The approximate coefficient has low frequency coefficients represent significant information similar to input signal or array. The detailed coefficients represent the detailed information represents high frequency coefficients. The $\mathrm{L}$ and $\mathrm{H}$ are computed using following illustration.

The row vector having four elements says $A$ is considered and is converted into DWT bands.
$\mathrm{A}=\left(\begin{array}{llll}1 & 2 & 3 & 4\end{array}\right)$

The Low (L) and High (H) frequency bands of first two elements of row vector $A$ are computed using equations 3 and 4 respectively.

$L=\frac{(a+b)}{\sqrt{2}}=\frac{(1+2)}{\sqrt{2}}=2.1213$ 
$H=\frac{(a-b)}{\sqrt{2}}=\frac{(1-2)}{\sqrt{2}}=-0.7071$

The low frequency band coefficient values are high compared to high frequency coefficient values; hence low frequency band has significant information. Similarly L and H bands of third and fourth elements of $A$ are 4.949747 and -0.7071 respectively. The $\mathrm{L}$ and $\mathrm{H}$ bands of row vector $A$ are

$\mathrm{L}=\left(\begin{array}{ll}2.12132 & 4.949747\end{array}\right) \quad \mathrm{H}=\left(\begin{array}{ll}-0.7071 & -0.7071\end{array}\right)$

The very low values of high frequency coefficients are converted into moderate value using Log $(1+\mathrm{H})$. The final DWT features are obtained by concatenating low and modified high frequency coefficients.

3.5 Matching Unit: The features of database are compared with test features using Euclidian Distance (ED) as given in equation 5.

$E D=\sqrt{\sum_{i=1}^{K}\left|\left(M_{i}-N_{i}\right)\right|^{2}}$

Where $\mathrm{K}=$ Total Number of features

$\mathrm{M}_{\mathrm{i}}=$ Feature coefficient values of database vectors

$\mathrm{Ni}_{\mathrm{i}}=$ Feature coefficient values of test vectors

3.6 Matching Fusion: The performance parameters of two methods using FFT and DWT features separately are fused using normalization fusion technique as given in equation 6 .

Fused Opt.TSR $=\frac{\text { ActualValueofopt.TSRof FFT }}{\text { ActualValueofOpt.TSRofDWT }} *(\max ($ opt.TSR $)$ DWT $)$

\section{ALGORITHM}

Problem Definition: The face recognition is used to identify a person in several applications. The face images are identified based on transform domain features and fusion techniques. The proposed algorithm is given Table 1.

Objectives: The face images are used to recognize persons efficiently and the objectives are

(i) To increase Total Success Rate (TSR) and Optimum TSR

(ii) To decrease Error Rate

(iii) To decrease computation time to extract features. 
Signal \& Image Processing : An International Journal (SIPIJ) Vol.8, No.4, August 2017

Table 1: Proposed Face recognition algorithm

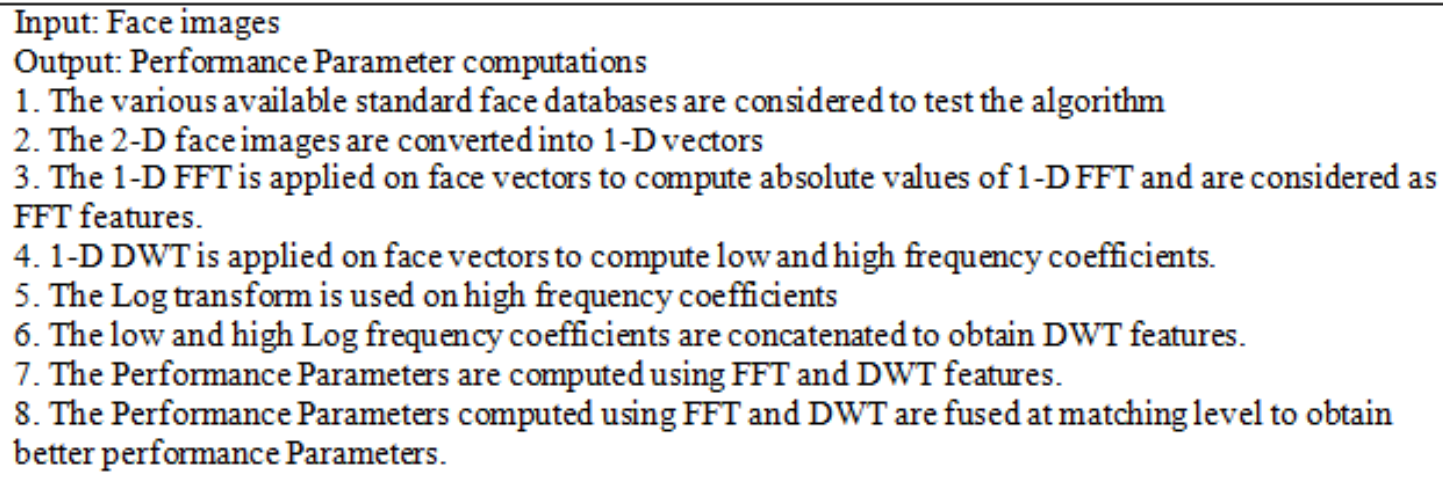

\section{Performance AnAlysis}

In this section, the definitions of performance parameters, result analysis using FFT, DWT and fusion techniques and comparison of proposed method with existing methods are discussed. The variations of Performance parameters of each database using different combinations of Person Inside Database (PID) and Person Outside Database (POD) are also discussed.

\subsection{Definitions of Performance Parameters:}

1. False Rejection Ratio (FRR): The ratio of the falsely rejected persons in the inside database to the total number of persons available in the database. The percentage of FRR is calculated using an equation 7.

$$
F R R=\frac{\text { Number of Geniue Persons rejected in the inside database }}{\text { Total Number of persons in the database }}
$$

2. False Acceptation Ratio (FAR): The ratio of the persons falsely accepted that are not in the database to the total number of persons considered from the outside database.

$$
F A R=\frac{\text { Number of Persons accepted from the outside database }}{\text { Total Number of persons in the outside database }}
$$

3. Total Success Rate (TSR): The ratio of total number of persons matched to the total number of persons in the database.

$$
T S R=\frac{\text { Number of Persons matched correctly }}{\text { Total Number of persons in the inside database }}
$$

4. Equal Error Rate (EER): The error rate where both the FAR and FRR are equal and less.

5. Half Error Rate (HER): The average of two errors FAR and FRR.

$$
H E R=\frac{F A R+F R R}{2}
$$


Signal \& Image Processing : An International Journal (SIPIJ) Vol.8, No.4, August 2017

5.2 Analysis of Results using FFT: The performance parameter variations for different face databases such as Indian Female, Indian Male, NIR, YALE and ORL using FFT technique are analyzed.

5.2.1 Indian Female Database: The performance parameters are varied with the reference to the threshold combinations of 7:15,11:11 and 15:7. The values of percentage FAR and TSR increase with threshold, where as the FAR values decreased with threshold. The graphical representation of performance parameters variations with threshold are shown in Figure 8,9 and 10 for PID and POD combinations of 7:15, 11:11 and 15:7. The percentage values of FAR and TSR are directly proportional to the threshold, where as the FRR values are inversely proportional to the threshold.

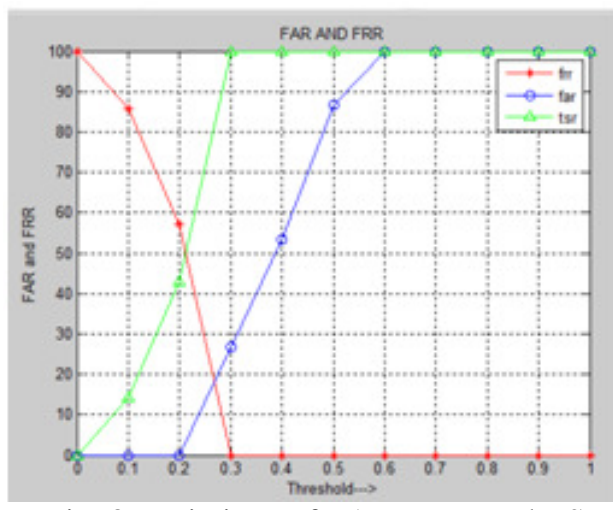

Fig. 8 Variations of FAR, FRR and TSR with PID: POD =7:15

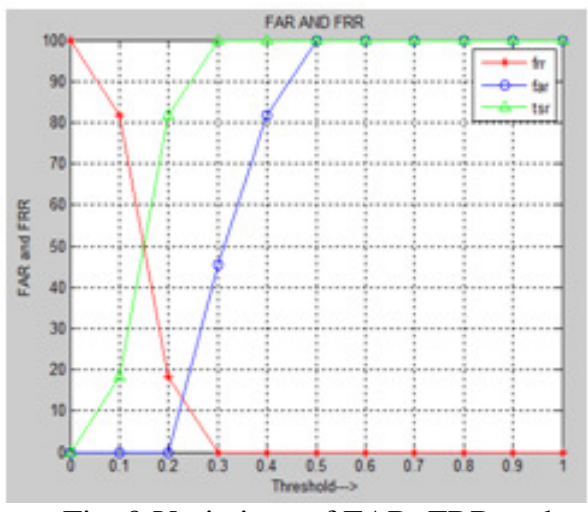

Fig. 9 Variations of FAR, FRR and TSR with PID: POD $=11: 11$

The percentage EER values are 19,13 and 15 for PID and POD combinations of 7:15, 11:11 and 15:7 respectively. The percentage opt.TSR values are 80,90 and 85 for PID and POD combinations of 7:15, 11:11 and 15:7 respectively.

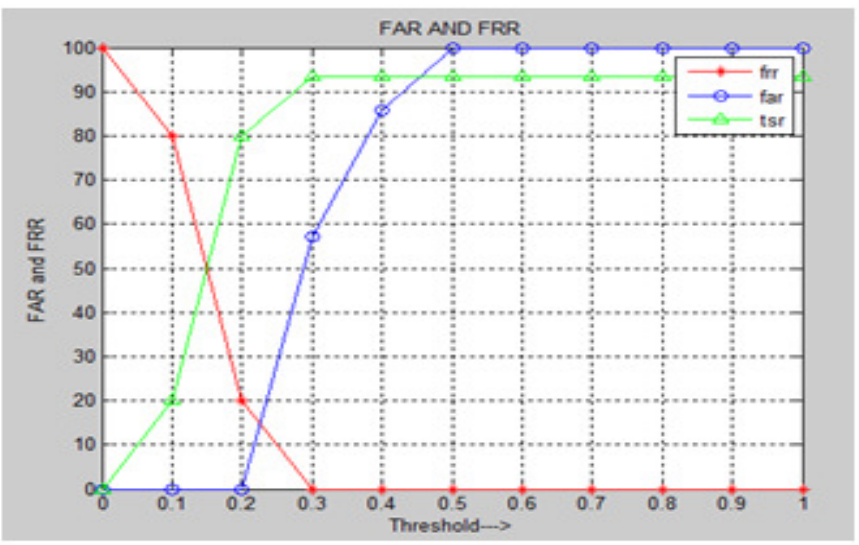

Fig. 10 Variations of FAR, FRR and TSR with PID: POD =15:7

5.2.2 Indian Male Database: The variations of performance parameter values with threshold for PID and POD combinations of 5:15, 10:10 and 15:5 are shown in figures 11, 12 and 13 respectively. The percentage EER values are for PID: POD combinations of 5:15, 10:10 and 15:5 are 18, 38 and 42 respectively. The percentage opt.TSR values are 80, 61 and 59 for PID and POD combinations of 5:15, 10:10 and 15:5 respectively. 
Signal \& Image Processing : An International Journal (SIPIJ) Vol.8, No.4, August 2017

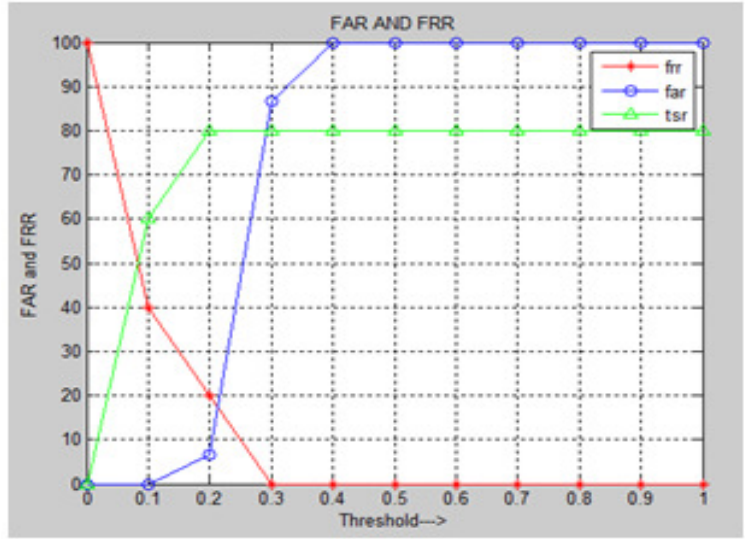

Fig. 11 Variations of FAR, FRR and TSR with PID: POD =5:15

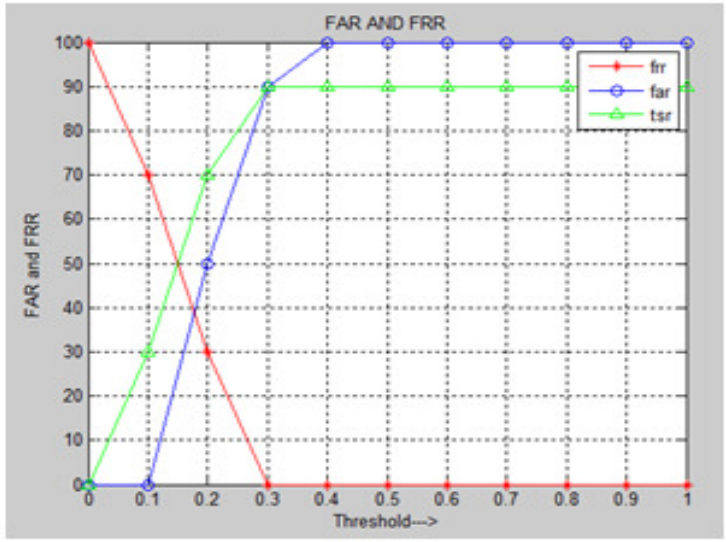

Fig. 12 Variations of FAR, FRR and TSR with PID: POD =10:10

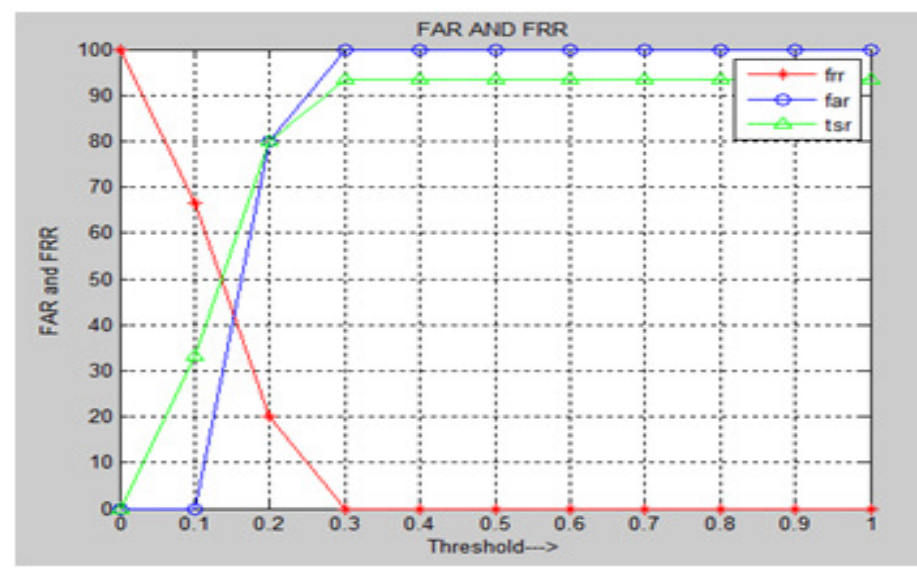

Fig. 13 Variations of FAR, FRR and TSR with PID: POD =15:5

5.2.3 NIR Database: The variations of percentage parameters such as percentage FRR, FAR and TSR with threshold for PID and POD combinations of 10:30, 20:20 and 30:10 are shown in Figures 14, 15 and 16.

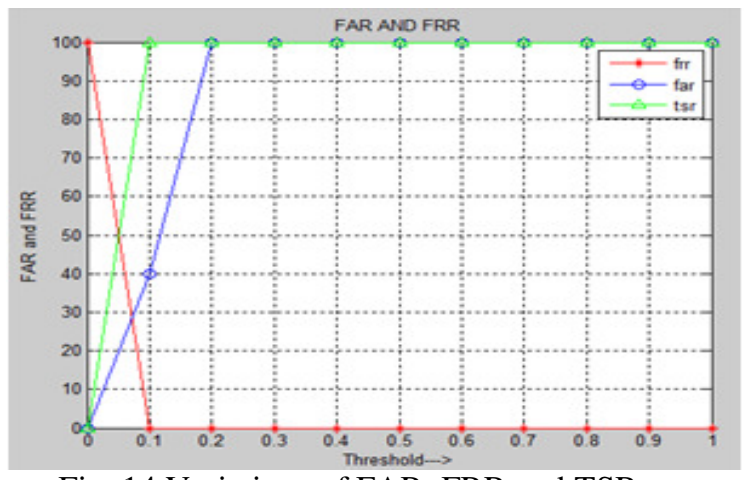

Fig. 14 Variations of FAR, FRR and TSR with PID: POD =10:30

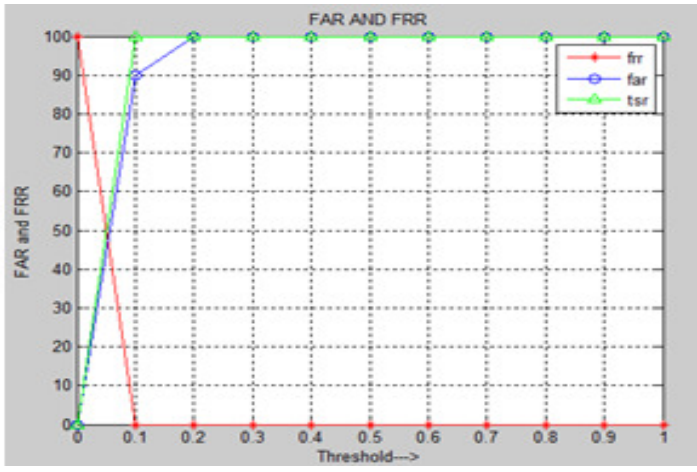

Fig. 15 Variations of FAR, FRR and TSR with PID: POD $=20: 20$ 
Signal \& Image Processing : An International Journal (SIPIJ) Vol.8, No.4, August 2017

The percentage EER values for PID and POD combinations of 10:30, 20:20 and 30:10 are 28, 48 and 17 respectively. The percentage opt.TSR values are 72, 52 and 80 for PID and POD combinations of 10:30, 20:20 and 30:10 respectively.

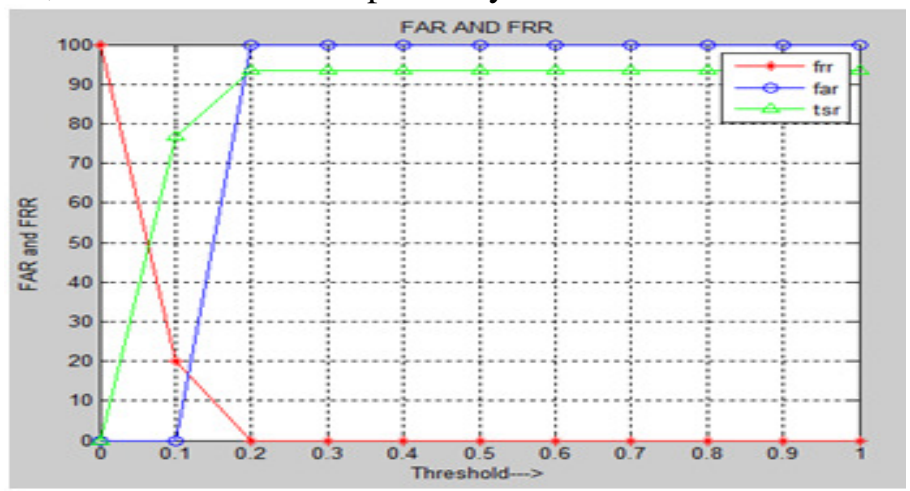

Fig. 16 Variations of FAR, FRR and TSR with PID: POD =30:10

5.2.4 YALE Database: The variations of percentage parameters such as percentage FRR, FAR and TSR with threshold for PID and POD combinations of 4:11, 8:7 and 11:4 are shown in Figures 17, 18 and 19.

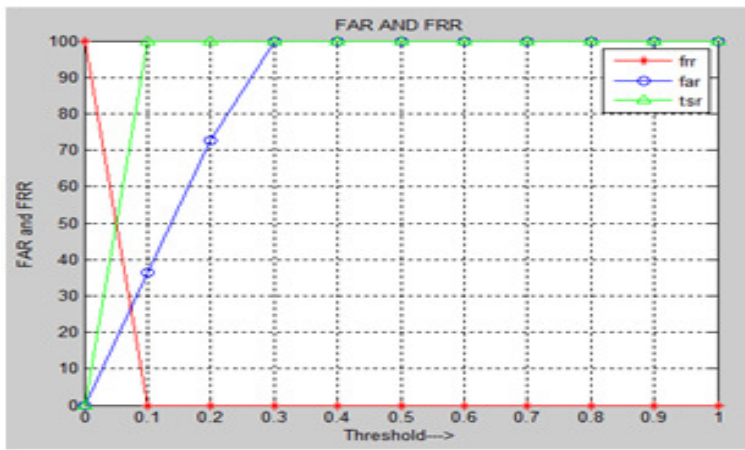

Fig. 17 Variations of FAR, FRR and TSR with PID: POD =4:11

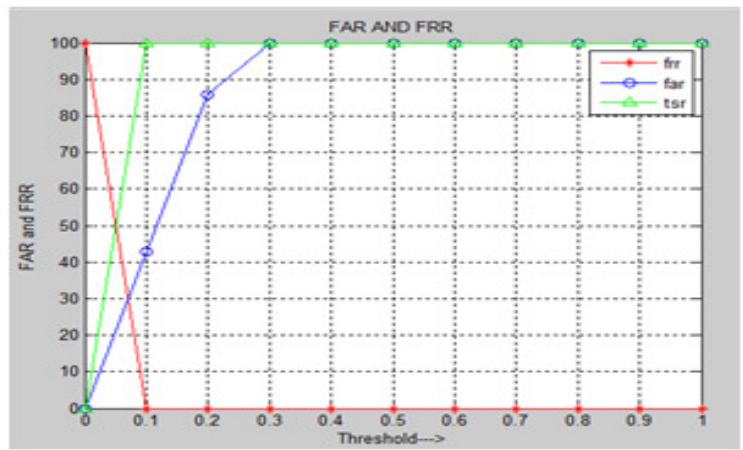

Fig. 18 Variations of FAR, FRR and TSR with PID: POD =8:7

The percentage EER values are 28,30 and 38 for PID and POD combinations of 4:11, 8:7 and 11:4 respectively. The corresponding percentage opt.TSR values are 82, 71 and 62 .

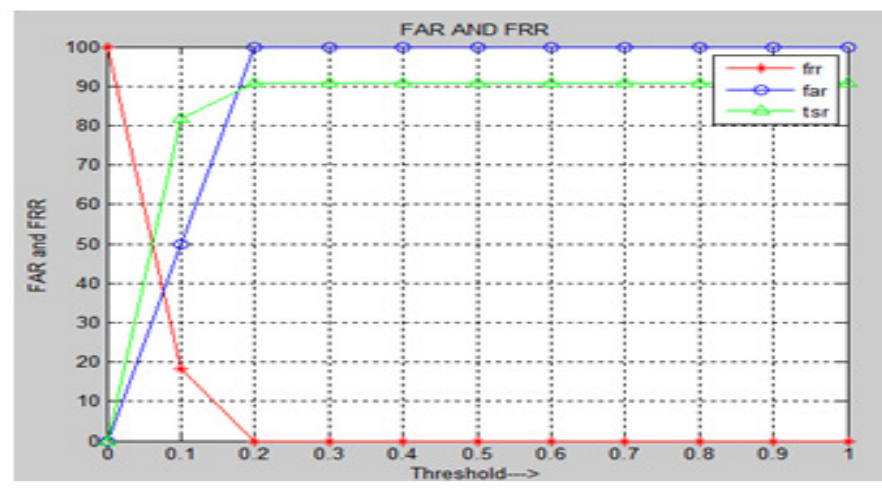

Fig. 19 Variations of FAR, FRR and TSR with PID: POD =11:4 
Signal \& Image Processing : An International Journal (SIPIJ) Vol.8, No.4, August 2017

5.2.5 ORL Database: The variations of percentage parameters such as percentage FRR, FAR and TSR with threshold for PID and POD combinations of 10:30, 20:20 and 30:10 are shown in Figures 20, 21 and 22.

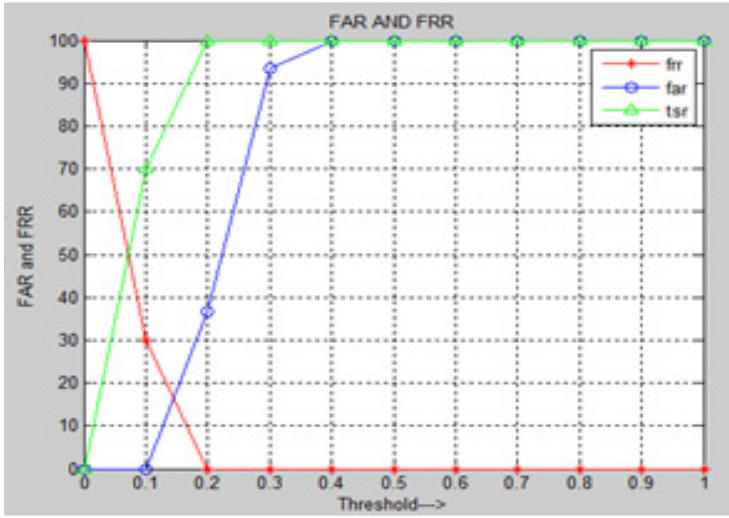

Fig. 20 Variations of FAR, FRR and TSR with PID: POD =10:30

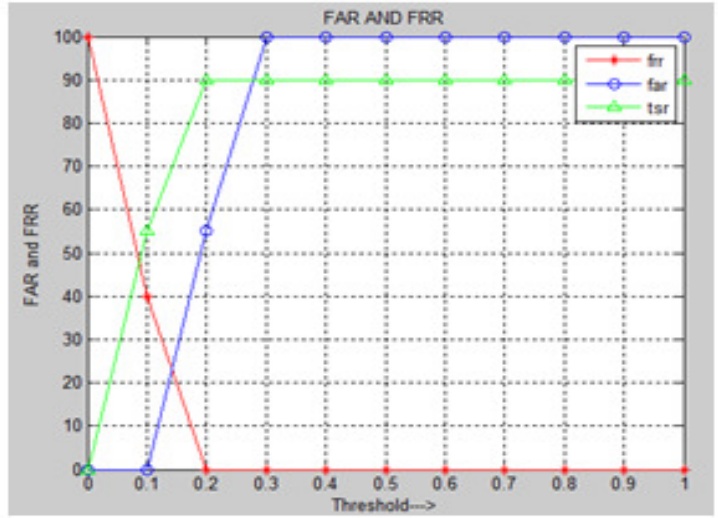

Fig. 21 Variations of FAR, FRR and TSR with PID: POD =20:20

The Percentage EER values are 18, 21 and 15 for PID and POD combination of 10:30, 20:20 and 30:10. The corresponding percentage opt.TSR values are 82, 69 and 82 respectively.

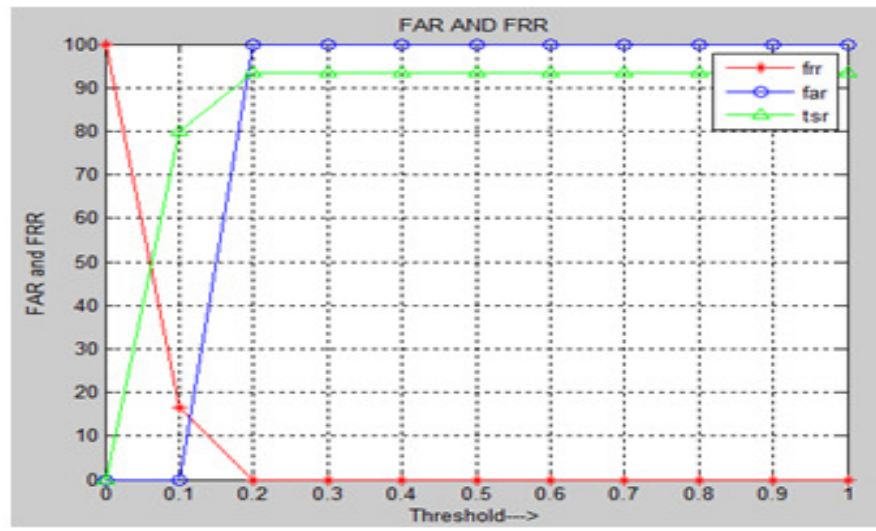

Fig. 22 Variations of FAR, FRR and TSR with PID: POD =30:10

5.3 Analysis of Results using DWT: The performance parameter variations for different face databases such as Indian Female, Indian Male, NIR, YALE and ORL using DWT technique are analyzed.

5.3.1 Indian Female Database: The performance parameters are varied with the reference to the threshold for various combinations of PID and POD. The percentage variation of FAR,FRR and TSR for PID and POD combinations of 7:15,11:11 and 15:7 are shown in Figure 23, 24 and 25. It is observed that the values of FAR and TSR are increases with threshold, whereas FRR decreases with threshold. The maximum TSR values for PID and POD combinations of 7:15, 11:11 and 15:7 are 85.71, 90.9 and 86.6 respectively. The optimum TSR values are 85.7, 82 and 72for PID and POD combination of 7:15,11:11 and 15:7 respectively and the corresponding EER values are 12,12 and 19. 
Signal \& Image Processing : An International Journal (SIPIJ) Vol.8, No.4, August 2017

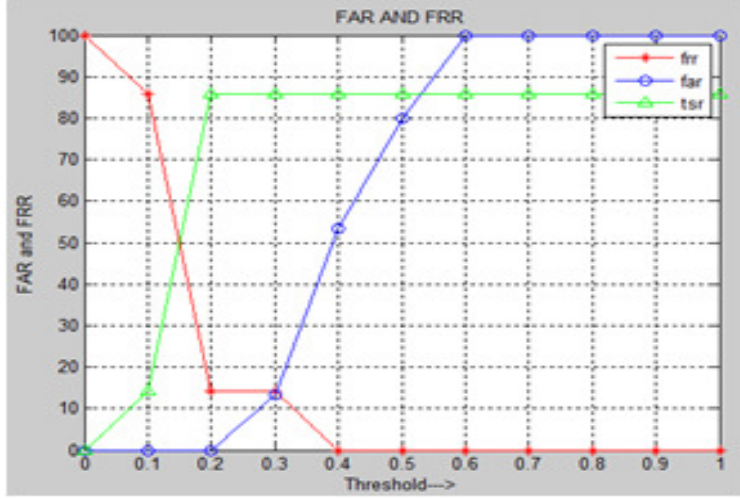

Fig. 23 Variations of FAR, FRR and TSR with PID: POD =7:15

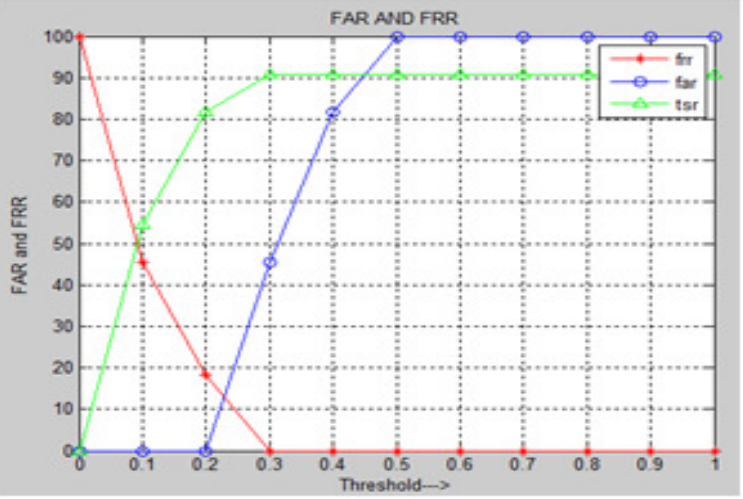

Fig. 24 Variations of FAR, FRR and TSR with PID: POD $=11: 11$

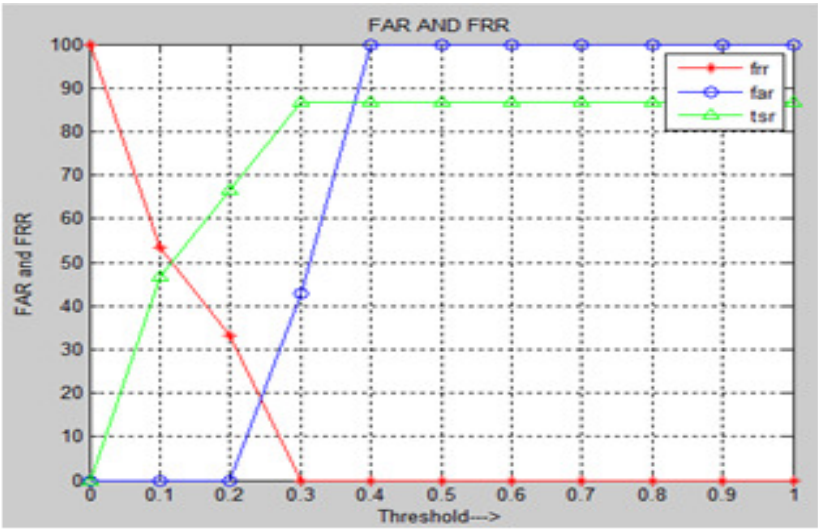

Fig. 25 Variations of FAR, FRR and TSR with PID: POD =15:7

5.3.2 Indian Male Database: The variations of performance parameter values with threshold for PID and POD combinations of 5:15, 10:10 and 15:5 are shown in figures 26, 27 and 28 respectively. The percentage EER values for PID: POD combinations of 5:15, 10:10 and 15:5 are 20,39 and 50 respectively and the corresponding percentage optimum TSR values are 80, 60 and 50 .

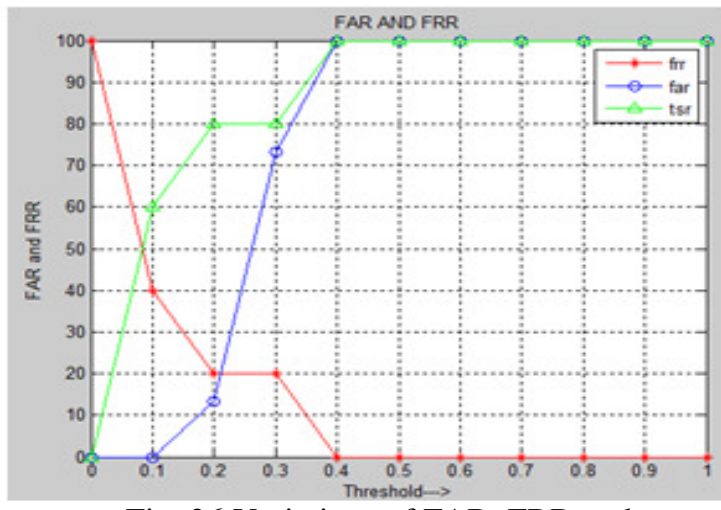

Fig. 26 Variations of FAR, FRR and TSR with PID: POD =5:15

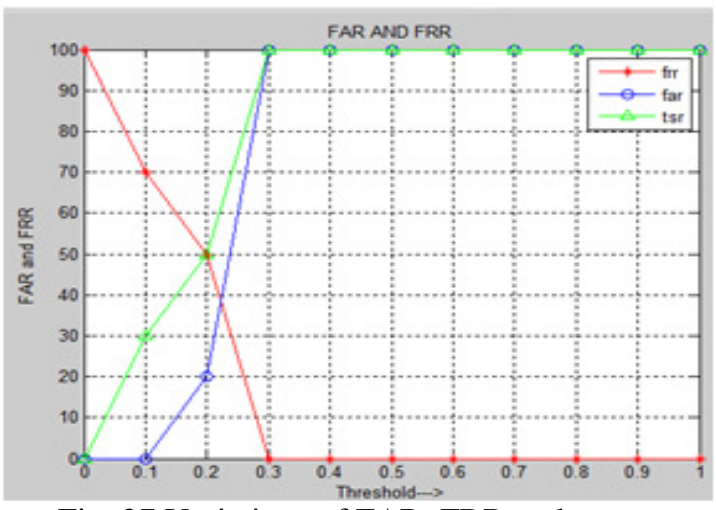

Fig. 27 Variations of FAR, FRR and TSR with PID: POD =10:10 
Signal \& Image Processing : An International Journal (SIPIJ) Vol.8, No.4, August 2017

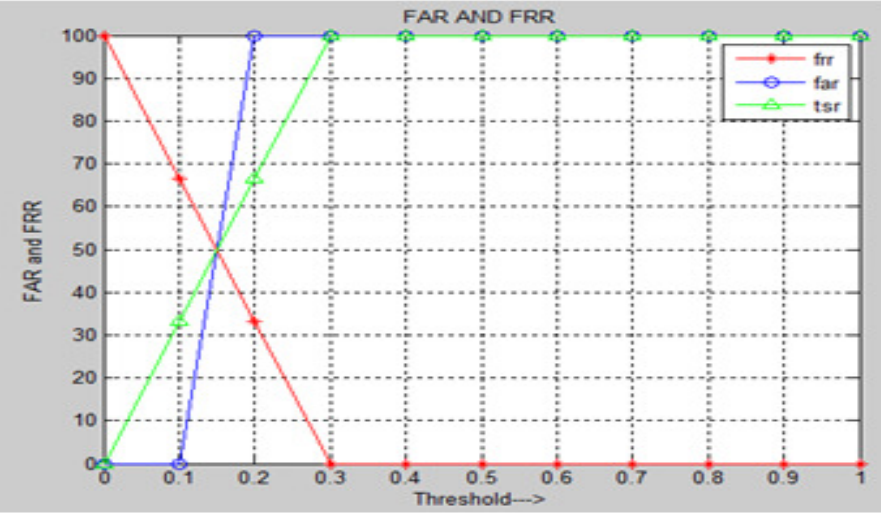

Fig. 28 Variations of FAR, FRR and TSR with PID: POD =15:5

5.3.3 NIR Database: The variations of Performance parameters such as percentage FRR, FAR and TSR with threshold for PID and POD combinations of 10:30, 20:20 and 30:10 are shown in Figures 29, 30 and 31.

The Percentage optimum TSR values are 72, 62 and 68 for PID and POD combination of 10:30, 20:20 and 30:10 respectively and also the corresponding percentage EER values are 19, 40 and 42.

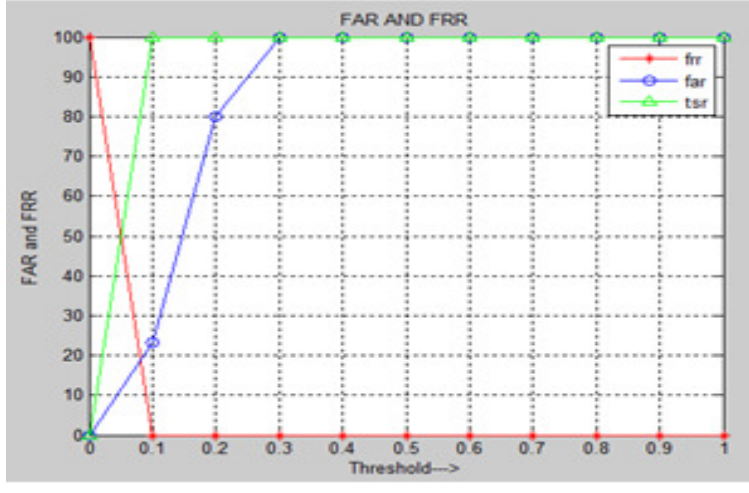

Fig. 29 Variations of FAR, FRR and TSR with PID: POD $=10: 30$

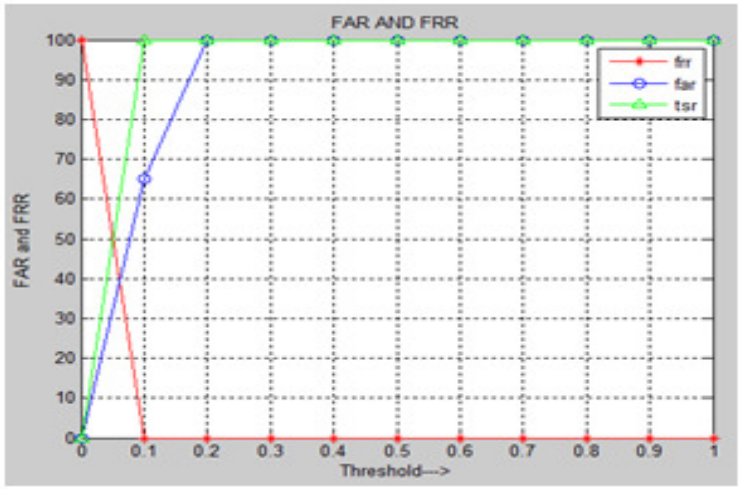

Fig. 30 Variations of FAR, FRR and TSR with PID: POD =20:20

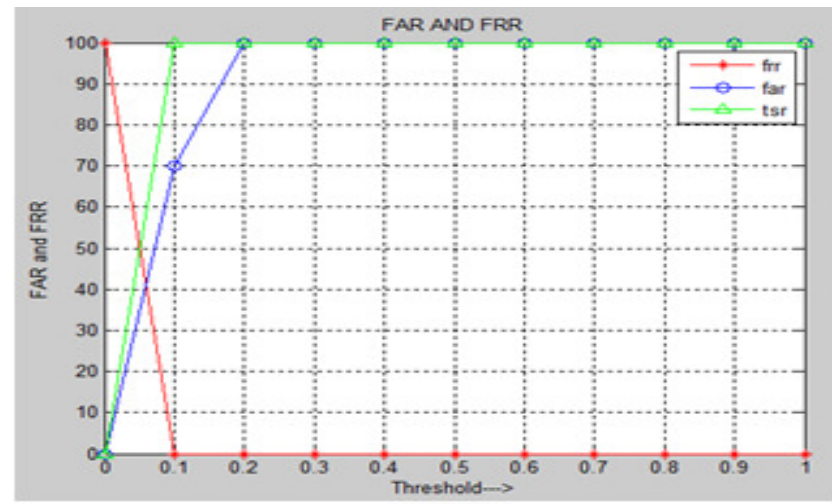

Fig. 31 Variations of FAR, FRR and TSR with PID: POD =30:10 
Signal \& Image Processing : An International Journal (SIPIJ) Vol.8, No.4, August 2017

5.3.4 ORL Database: The variations of Performance parameters such as percentage FRR, FAR and TSR with threshold for PID and POD combinations of 10:30, 20:20 and 30:10 are shown in Figures 32, 33 and 34. It is observed that the maximum percentage FAR and FRR values are 100 for all combination of PID and POD. The percentage optimum TSR values are 90, 80 and 72 for PID and POD combinations of 10:30, 20:20 and 30:10 respectively and also the corresponding percentage EER values are 10, 14 and 22 .

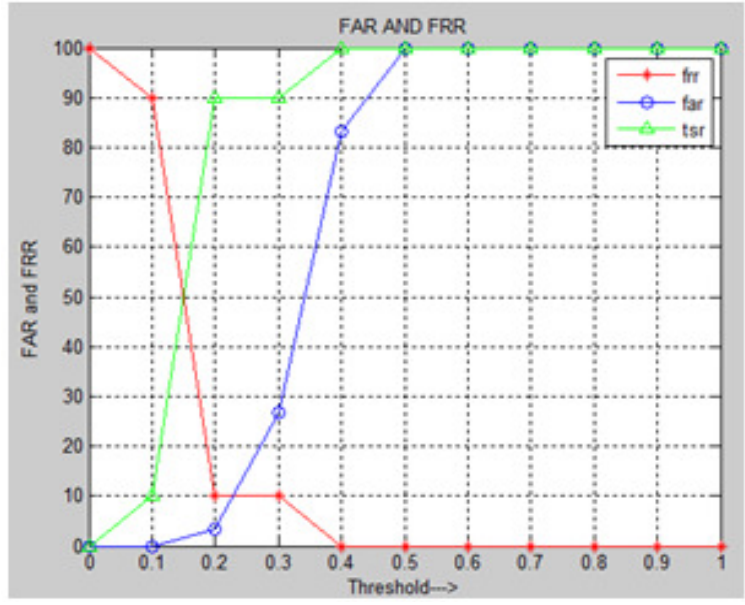

Fig. 32 Variations of FAR, FRR and TSR with PID: POD =10: 30

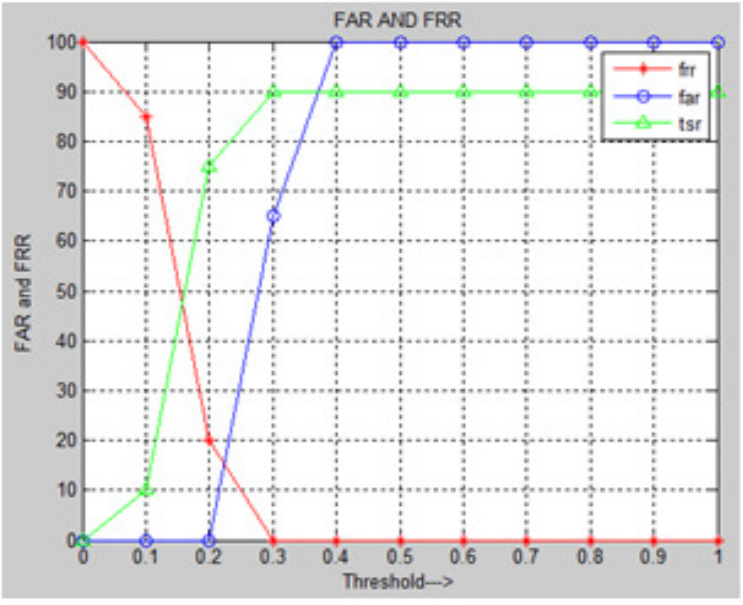

Fig. 33 Variations of FAR, FRR and TSR with PID: POD =20:20

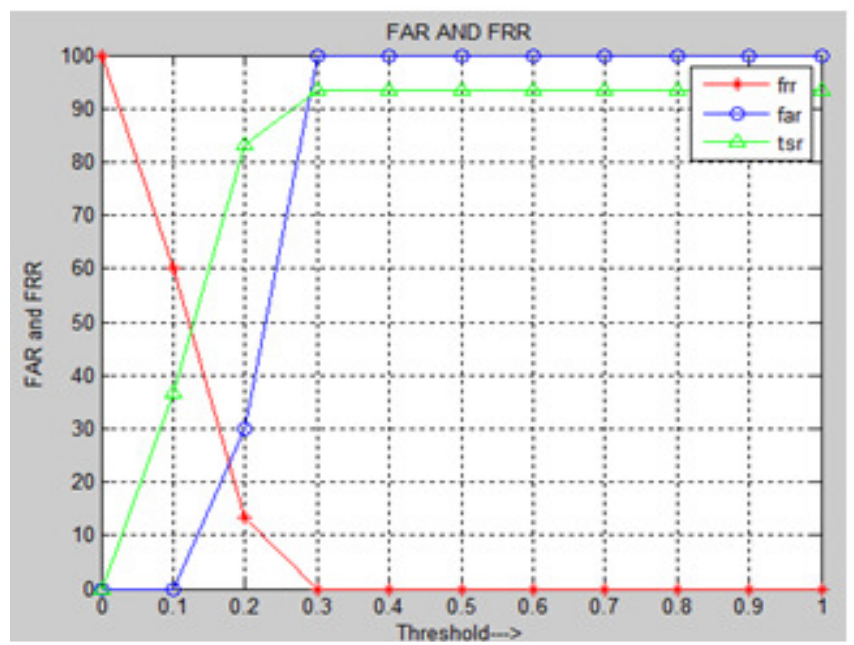

Fig. 34 Variations of FAR, FRR and TSR with PID: POD =30:10

5.3.5 YALE Database: The variations of performance parameters such as percentage FRR, FAR and TSR with threshold for PID and POD combinations of 4:11, 8:7 and 11:4 are shown in Figures 35, 36 and 37. The percentage maximum optimum TSR values are 75, 90 and 80 for PID and POD combinations of 4:11, 8:7 and 11:4 respectively and also the corresponding percentage EER values are 25, 10 and 12 . 
Signal \& Image Processing : An International Journal (SIPIJ) Vol.8, No.4, August 2017

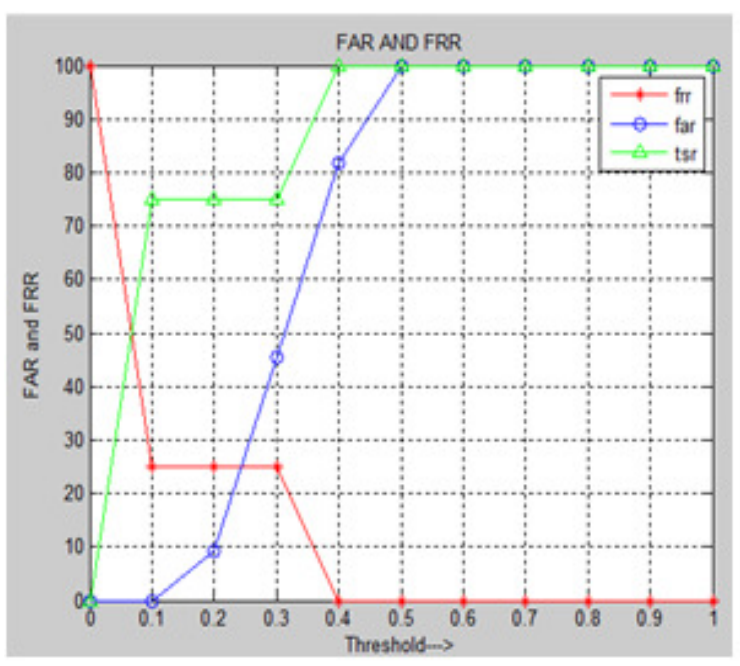

Fig. 35 Variations of FAR, FRR and TSR with PID: POD =4:11

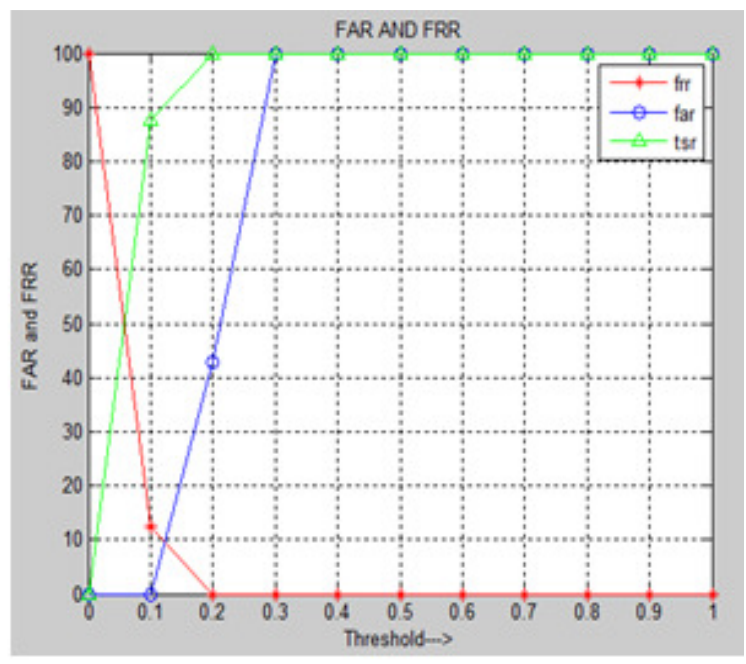

Fig. 36 Variations of FAR, FRR and TSR with PID: POD $=8: 7$

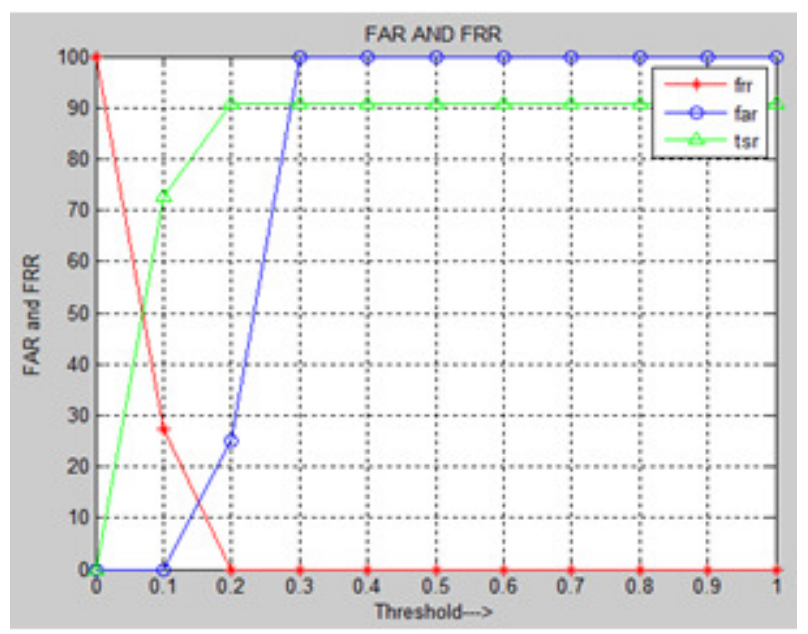

Fig. 37. Variations of FAR, FRR and TSR with PID: POD $=11: 4$

\subsection{Performance Comparison:}

The performance of proposed technique is compared with existing techniques such as FFT and DWT. The face databases such as ORL, Indian Male, Indian Female and Yale are used with different combinations of PID and PODs. The performance parameters are compared and are given in Table 2. The values of performance parameters are based on types of face databases used to test the algorithm.

The Percentage optimum recognition rate is high with YALE face database, whereas the recognition rate is low with NIR database. It is observed that, the proposed fusion technique is better compared to existing techniques in terms of recognition rate and error rate. 
Signal \& Image Processing : An International Journal (SIPIJ) Vol.8, No.4, August 2017

Table 2: Performance comparison of proposed method with existing techniques for ORL, Indian Male, Indian Female and YALE Face Database

\begin{tabular}{|c|c|c|c|c|c|c|c|c|c|c|}
\hline \multirow{2}{*}{$\begin{array}{l}\text { Data } \\
\text { bases }\end{array}$} & \multirow{2}{*}{$\begin{array}{l}\text { PID: } \\
\text { POD }\end{array}$} & \multicolumn{3}{|c|}{ FFT } & \multicolumn{3}{|c|}{ DWT } & \multicolumn{3}{|c|}{ Fusion } \\
\hline & & $\begin{array}{l}\text { \%Opt } \\
\text {.TSR }\end{array}$ & \%EER & \%Max.TSR & $\begin{array}{l}\text { \%Opt } \\
\text {.TSR }\end{array}$ & \%EER & \%Max.TSR & $\begin{array}{l}\text { \% Opt. } \\
\text { TSR }\end{array}$ & \%EER & \%Max.TSR \\
\hline \multirow[t]{3}{*}{$\overline{\text { ORL }}$} & $10: 30$ & 82 & 18 & 100 & 90 & 10 & 100 & 82 & 18 & 100 \\
\hline & $20: 20$ & 69 & 21 & 90 & 80 & 14 & 90 & 69 & 15 & 100 \\
\hline & $30: 10$ & 82 & 15 & 93.33 & 72 & 22 & 93.33 & 90 & 10 & 100 \\
\hline \multirow{3}{*}{$\begin{array}{l}\text { Indian } \\
\text { Female }\end{array}$} & $7: 15$ & 80 & 19 & 100 & 85.71 & 12 & 85.71 & 80 & 19 & 100 \\
\hline & $11: 11$ & 90 & 13 & 100 & 82 & 12 & 90.909 & 87.80 & 12 & 94.28 \\
\hline & $15: 7$ & 85 & 15 & 93.33 & 72 & 19 & 86.667 & 97.61 & 8.21 & 92.29 \\
\hline \multirow{3}{*}{$\begin{array}{l}\text { Indian } \\
\text { Male }\end{array}$} & $5: 15$ & 80 & 18 & 80 & 80 & 20 & 100 & 80 & 18 & 80 \\
\hline & $10: 10$ & 61 & 38 & 90 & 60 & 39 & 100 & 81.33 & 20 & 90 \\
\hline & $15: 5$ & 59 & 42 & 93.33 & 50 & 50 & 100 & 94.40 & 16.4 & 93.33 \\
\hline \multirow[t]{3}{*}{ NIR } & $10: 30$ & 72 & 28 & 100 & 72 & 19 & 100 & 72 & 19 & 100 \\
\hline & $20: 20$ & 52 & 48 & 100 & 62 & 40 & 100 & 60.38 & 22.56 & 100 \\
\hline & $30: 10$ & 80 & 17 & 93.3 & 68 & 42 & 100 & 61.00 & 21.46 & 100 \\
\hline \multirow[t]{3}{*}{ YALE } & $4: 11$ & 82 & 28 & 100 & 75 & 25 & 100 & 98.4 & 11.2 & 100 \\
\hline & $8: 7$ & 71 & 30 & 100 & 90 & 10 & 100 & 81 & 30 & 100 \\
\hline & $11: 4$ & 62 & 38 & 90.909 & 80 & 12 & 90.909 & 69.69 & 31.625 & 100 \\
\hline
\end{tabular}

\subsection{Performance comparison of proposed method with existing methods:}

The performance of the proposed method is compared with existing methods in terms of percentage optimum TSR (\%Opt. TSR) and EER are given in Table 3 for different face databases.

Table 3: Comparison of proposed method with existing methods

\begin{tabular}{|l|l|l|l|l|l|l|l|}
\hline Authors & Technique & \multicolumn{2}{|c|}{ Indian Male } & \multicolumn{2}{|c|}{ Indian Female } & \multicolumn{2}{c|}{ ORL } \\
\cline { 3 - 8 } & & $\begin{array}{l}\text { \%Opt. } \\
\text { TSR }\end{array}$ & \%EER & $\begin{array}{l}\text { \%Opt. } \\
\text { TSR }\end{array}$ & \%EER & $\begin{array}{l}\text { \%Opt. } \\
\text { TSR }\end{array}$ & \%EER \\
\hline $\begin{array}{l}\text { Sujatha BM, et } \\
\text { al., [19] }\end{array}$ & $\begin{array}{l}\text { Reversible Logic } \\
\text { Multiplier for Canny Edge }\end{array}$ & 70 & 32 & 80 & 19 & 50 & 50 \\
\hline $\begin{array}{l}\text { Sujatha BM, et } \\
\text { al.,[20] }\end{array}$ & DWT+LBP+SOM & 55 & 40 & 72 & 30 & 60 & 30 \\
\hline $\begin{array}{l}\text { Sujatha BM, et } \\
\text { al., [21] }\end{array}$ & SVM+DWT & 58.33 & 44 & 87.5 & 12.5 & 90 & 10 \\
\hline Proposed & $\begin{array}{l}\text { Mean+DWT+FFT+ } \\
\text { Fusion }\end{array}$ & $\mathbf{9 4 . 4 0}$ & $\mathbf{1 6 . 4}$ & $\mathbf{9 7 . 6 1}$ & $\mathbf{8 . 2 1}$ & $\mathbf{9 0}$ & $\mathbf{1 0}$ \\
\hline
\end{tabular}

The Indian Male, ORL and Indian Female and ORL face databases are used to compare performance parameters of proposed method with existing methods presented by Sujatha et al., $[19,20,21]$. The performance values of Opt. TSR are high in the case of proposed method compared to existing methods. The percentage EER values are less in the case of proposed method compared to existing methods for all face databases. The performance parameters such as percentage recognition rate and half error rate of proposed method are compared with existing method presented by Belahcene, et al., [22] using Yale database is given in Table 34. It is observed that the performance of proposed method is better compared to existing method. 
Signal \& Image Processing : An International Journal (SIPIJ) Vol.8, No.4, August 2017

Table 34: Performance parameters comparison of proposed method with existing methods using Yale Database

\begin{tabular}{|l|l|l|l|}
\hline Authors & Techniques & \%Recognition Rate & \%Half Error Rate \\
\hline Belahcene, et al., [22] & TLPP & 73.33 & 15.00 \\
\hline Belahcene, et al., [22] & BSIF+TLPP (7x7) & 75.56 & 15.87 \\
\hline Proposed & Mean+DWT+FFT+Fusion & $\mathbf{9 8 . 4}$ & $\mathbf{3 . 0 0}((\mathbf{6 + 0}) / \mathbf{2})$ \\
\hline
\end{tabular}

The performance of proposed method is better for the following reasons

i. The 2D face images are converted into 1D vector to reduce number of features without disturbing much of significant information in the face images to reduce complexity of an algorithm.

ii. The 1D-FFT and DWT features are better compared to spatial domain features.

iii. The computed performance parameters of FFT and DWT are fused at the matching level using normalization fusion technique.

iv. The results obtained in the proposed technique support the real time implementation by increasing speed of computation.

\section{CONCUSION}

The face is a physiological biometric traits used to recognize a person and used in several applications.

In this paper, Compression based Face Recognition using Transform Domain Features fused at Matching Level is proposed. The transform domain techniques such as 1D- FFT and 1D- DWT are used to extract initial features of face images by converting 2D into 1D. The ED is used to compare initial features of database images and test images to compute initial performance parameters. The final performance parameters are obtained by fusing initial performance parameters at matching level for better results. It is observed that, the performance of the proposed method is better compared to existing methods. In future, the proposed method will be implemented using hardware for real time applications.

\section{REFERENCE}

[1] Ramy C.G.Chehata , Wasfy B. Mikhael and Moataz M. Abdelwahab , "Transform Domain Two Dimensional and Diagonal Modular Principal Component Analysis for Facial Recognition Employing Different Windowing Techniques", IEEE International Midwest Symposium on Circuits and Systems, pp 1104-1107, 2013.

[2] Md. Iqbal Quraishi, Goutam Das, Arindam Das, Poulami Dey and Amara Tasneem, "A Novel Approach for Face Detection using Artificial Neural Network", International Conference on Intelligent Systems and Signal Processing, pp 179-184, 2013.

[3] Jing Wang, Canyi Lu, Meng Wang, Peipei Li, Shuicheng Yan, and Xuegang Hu, "Robust Face Recognition via Adaptive Sparse Representation", IEEE Transactions on Cybernetics, Vol: 44, Issue: 12, pp 2368 - 2378, December2014. 
Signal \& Image Processing : An International Journal (SIPIJ) Vol.8, No.4, August 2017

[4] Zhao-Rong Lai, Dao-Qing Dai, Chuan-Xian Ren and Ke-Kun Huang, "Discriminative and Compact Coding for Robust Face Recognition", IEEE Transactions on Cybernetics, Vol: 45, Issue: 9, pp 1900 - 1912,September 2015

[5] Dmitry Gorodnichy and Eric Granger, "Target-Based Evaluation of Face Recognition Technology for Video Surveillance Applications", IEEE Symposium on Computational Intelligence in Biometrics and Identity Management, 2014

[6] Yi Jin, Jiwen Lu, and QiuqiRuan, "Coupled Discriminative Feature Learning for Heterogeneous Face Recognition”, IEEE Transactions on Information Forensics and Security, Vol. 10, no. 3, pp 640-652, March 2015

[7] Asem M. Ali, "A 3D-Based Pose Invariant Face Recognition at a Distance Framework", IEEE Transactions on Information Forensics and Security, Vol. 9, no. 12, pp 2158-2169, December 2014.

[8] Priyanka Dharani, A S Vibhute, "Face Recognition Using Wavelet Neural Network", International Journal of Advanced Research in Computer Science and Software Engineering, Vol 7, Issue 5, pp 101-107, May 2017.

[9] Lihong Wan, Na Liu, Hong Huo and Tao Fang, "Face Recognition with Convolutional Neural Networks and Subspace Learning", International Conference on Image, Vision and Computing, pp 228-233, July 2017.

[10] Pawanpreet KaurHarra, Deepak Aggarwal, "Hybrid Approach for Face Recognition Using DWT and LBP" International Journal on Recent and Innovation Trends in Computing and Communication Vol. 5 no. 7

[11] Kumuda T and L Basavraj "Edge Based Segmentation Approach to Extract Text from Scene Images" IEEE International Advance Computing Conference, pp 706-710, January 2017.

[12] Hameed R. Farhan, Mahmuod H. Al-Muifraje and Thamir R. Saeed "A Novel Face Recognition Method based on One State of Discrete Hidden Markov Model" conference on New Trends in Information \& Communications Technology Applications, pp 252-257, March 2017.

[13] The ORL database, http://www.cam-orl.co.uk

[14] The NIR database, http://www.cbsr.ia.ac.cn/english/NIR-VIS-2.0-Database.html

[15] Yale database, http://vision.ucsd.edu/content/yale-face-database

[16] Indian Face Database, http://viswww.cs.umass.edu/ vidit/Indian Face Database

[17] Sujatha BM , K Suresh Babu , Raja K B and Venugopal K R, "Optimized Face Recognition Algorithm using Spatial and Transform Domain Techniques", International Conference on Image Information Processing,pp190-195, 2015.

[18] Dipalee Gupta and Siddhartha Choubey, "Discrete Wavelet Transform for Image Processing" International Journal of Emerging Technology and Advanced Engineering, Vol 4, Issue 3, pp 598602, March 2015.

[19] Sujatha B M, ShubhangiLagali, NayinaRamapur, K Suresh Babu, K B Raja and Venugopal K R, "Reversible Logic-MUX-Multiplier Based Face Recognition using Hybrid Features", IOSR Journal of VLSI and Signal Processing, Vol.6, No. 06, pp 48-64, December 2016. 
Signal \& Image Processing : An International Journal (SIPIJ) Vol.8, No.4, August 2017

[20] Sujatha B M, NayinaRamapur, ShubhangiLagali, K Suresh Babu, K B Raja and Venugopal K R,"SOM based Face Recognition using Steganography and DWT Compression Techniques", International Journal of Computer Science and Information Security, Vol. 14 No. 9 September 2016 pp 806-826.

[21] Sujatha B M, ChetanTippannaMadiwalar, Suresh Babu K, Raja K B and Venugopal K R, "Compression Based Face Recognition Using DWT and SVM", Signal \& Image Processing : An International Journal (SIPIJ) Vol.7, No.3, pp 45-62, June 2016 .

[22] M.Belahcene1, M.Laid, A.Chouchane, A.Ouamane and S.Bourennane, "Local Descriptors and Tensor Local Preserving Projection in Face Recognition", Visual Information Processing (EUVIP), 2016 6th European Workshop Oct. 2016. 